\title{
Contribuição para o diagnóstico de peste
}

\author{
Contribution towards plague diagnosis
}

\author{
Alzira Maria Paiva de Almeida ${ }^{1}$, Gerlane Tavares de Souza ${ }^{1}$, Silvana Santos ${ }^{1}$, \\ Soraya Cavalcante Silva ${ }^{1}$, Marina de Moraes Vasconcelos Petribú ${ }^{1}$, \\ Patrícia de Oliveira Haver $^{1}$, Antônia Ivoneida Aragão ${ }^{2}$ e Celso Tavares ${ }^{3}$
}

\begin{abstract}
RESUMO
Apesar de sua fundamentação clínico-epidemiológica, numerosos casos suspeitos de peste nos focos brasileiros têm sido descartados por serem negativos pelo teste de hemaglutinação para detecção de anticorpos contra o antígeno F1 da Yersinia pestis. A transcendência da peste justifica estudar se tais resultados decorrem da falta de resposta ao F1, e se outras proteínas da Yersinia pestis poderiam ser reconhecidas nos soros suspeitos, sendo desta forma candidatas como alvo diagnóstico alternativo ao F1. Assim sendo, cepas de Yersinia pestis e de Yersinia pseudotuberculosis, uma proteína YopH recombinante e a F1 foram utilizadas para analisar soros de pacientes e soros imunes de coelhos. A F1 e a YopH não foram reconhecidas pelos soros bumanos HA- e nenhuma proteína majoritária, comum a todos os soros humanos e coelhos, foi identificada, o que permite concluir que os casos suspeitos devem ser submetidos a uma avaliação clínico-laboratorial mais rigorosa, aprofundando a investigação epidemiológica em busca de outras etiologias.
\end{abstract}

Palavras-chaves: Yersinia pestis. Peste. Antígeno F1. Proteína YopH. Diagnóstico. Vigilância epidemiológica.

\section{ABSTRACT}

Despite the clinical-epidemiological features of plague, numerous suspected cases in Brazilian outbreaks have been discarded because of negative results from the hemagglutination test for antibodies against the Yersinia pestis F1 antigen. The transcendence of plague justifies studying whether such results are due to unresponsiveness to F1, and whether other $\mathrm{Y}$. pestis proteins might be recognized in suspect serum. These would therefore be candidates to be alternative diagnostic targets to the F1 antigen. Thus, strains of Y. pestis and Y. pseudotuberculosis, a recombinant YopH protein and the F1 antigen were used to analyze serum from patients and immune serum from rabbits. F1 and YopH were not recognized by HA-negative buman serum and no major protein common to all the human and rabbit serum samples was identified. This allows the conclusion that suspected cases must be subjected to more rigorous clinical-laboratory evaluation, with strengthening of epidemiological investigations in the search of other etiologies.

Key-words: Yersinia pestis. Plague. F1 antigen. YopH protein. Diagnosis. Epidemiological surveillance.

A peste, infecção pela Yersinia pestis, mantém-se em vários focos naturais na África, Ásia e Américas e atualmente é considerada uma doença reemergente pela Organização Mundial da Saúde ${ }^{1524}$, constituindo um problema de Saúde Pública. A sua epidemiologia é bastante complexa e a erradicação ainda não é exeqüível, apesar dos avanços científicos e tecnológicos ${ }^{13}$. 0 seu potencial de epidemização e elevada letalidade justificam a sua inclusão na Classe I do Regulamento Sanitário Internacional (RSI) vigente, que exige notificação compulsória de toda atividade pestosa e manutenção de vigilância permanente nos focos e locais por onde a infecção possa ser introduzida a partir de focos ativos.

Vários fatores de virulência da $Y$. pestis são codificados em genes localizados no cromossomo e nos três plasmídeos prototípicos: pPst, pFra e pYV. 0 plasmídeo pPst, específico da Y.pestis, codifica uma protease (Pla) ou ativador do plasminogênio. No plasmídeo pFra, também específico, estão localizados os genes que codificam uma proteína de envoltório, a fração antigênica F1,

1. Departamento de Microbiologia do Centro de Pesquisas Aggeu Magalhães, Recife, PE. 2. Secretaria Estadual de Saúde, Fortaleza, CE. 3. Departamento de Clínica Médica da Faculdade de Medicina da Universidade Federal de Alagoas, Maceió, AL.

Apoio financeiro: PAPES-FIOCRUZ, SVS/MS e CNPq.

Endereço para correspondência: Dra Alzira de Almeida. CPqAM/FIOCRUZ. Campus da UFPE, Cidade Universitária, 50670-420 Recife, PE.

Tel: 5581 2101-2634; Fax: 5581 3453-2449.

e-mail: aalmeida@cpqam.fiocruz.br

Recebido para publicação em 30/5/2006

Aceito em 17/1/2007 
e a toxina murina ${ }^{19}$. A F1 é imunogênica para homens e animais, 0 que fundamenta a sua utilização na maioria dos testes diagnósticos da peste 9 . A toxina murina (Ymt) é uma exotoxina que parece desempenhar um importante papel na colonização do estômago das pulgas pela $Y$. pestis ${ }^{16}$, é menos imunogênica que a F1 e o seu emprego no diagnóstico sorológico é irrelevante ${ }^{18}$. 0 plasmídeo pYV é indispensável para a virulência da bactéria e codifica um complexo sistema de secreção tipo III, responsável pela síntese de várias proteínas (Yops) envolvidas na neutralização das defesas dos hospedeiros, sendo também encontrado nas outras yersínias patogênicas $^{10}$. 0 uso das Yops no diagnóstico da peste limita-se a uns poucos testes ${ }^{1417}$.

A prova da hemaglutinação (HA) com hemácias de carneiro para detecção de anticorpos contra 0 antígeno F1 é utilizada há décadas no diagnóstico e vigilância da peste ${ }^{23}$. As amostras pareadas de soro, colhidas na fase aguda e na convalescença, devem apresentar uma diferença de quatro títulos ou mais. A soroconversão ocorre uma a duas semanas após o início dos sintomas na maioria dos pacientes, mas pode ser mais precoce ou também não ocorrer ${ }^{1345}$.

A plasticidade do genoma da $Y$. pestis, com perda de plasmídeos, de segmentos cromossômicos e inserção de sequiências ${ }^{19}$, permite supor que a falta de resposta de alguns casos poderia decorrer de infecções por cepas atípicas não produtoras de F1, o que implicaria ocorrência de casos falso-negativos. Cumpre, pois, identificar novos alvos, o que possibilitará 0 desenvolvimento de uma nova geração de técnicas diagnósticas baseadas em outras características da bactéria.

0 objetivo deste trabalho foi verificar se outras proteínas da $Y$. pestis são reconhecidas pelos soros reagentes e não reagentes nos testes de detecção de anticorpos anti-F1, base do diagnóstico da peste no homem e em animais, identificando possíveis alvos para o desenvolvimento de novos testes diagnósticos mais sensíveis e específicos.

\section{MATERIAL E MÉTODOS}

Soros de coelhos imunizados, segundo Bahmanyar \& Cavanaugh ${ }^{6}$, com uma cepa de $Y$. pseudotuberculosis (IP 32950), três cepas de $Y$. pestis originárias de três focos pestosos brasileiros (P. Exu 769, P. PB 881 e P. CE 882) e duas cepas de referência estrangeiras (A1122 e EV76); 34 soros humanos (04 HA+) da soroteca do Serviço de Referência em Peste (SRP) do CPqAM; soro normal de coelho (SNC) e soro de coelho anti-F1 obtido segundo $\mathrm{Chu}^{9}$ foram analisados por Western-blot com proteínas totais das culturas bacterianas e com o antígeno F1, conforme protocolo descrito por Chu' 0 antígeno F1 foi extraído da cepa A1122 no SRP, de acordo com a técnica descrita por Chu?

278 soros de pacientes suspeitos de peste colhidos na rotina do Programa de Vigilância e Controle de Peste (PCP) no Estado do Ceará (CE) foram analisados por $\mathrm{HA}^{23}$ e Dot-Elisa ${ }^{2}$ Para confirmação desses resultados 15 amostras negativas (HA-) foram submetidas à HA e Competitive ELISA ${ }^{9}$ no Diagnostic and Reference Laboratory, Bacterial Zoonoses Branch, Division of
Vector-Borne Infectious Diseases, Centers for Disease Control and Prevention (DVBID/CDC, Fort Collins, CO/USA).

Para avaliar o potencial da proteína YopH recombinante no diagnóstico da peste, membranas contendo proteínas totais da cultura E. coli $\mathrm{DH} 5 \alpha$ transformada com o plasmídeo PTZ/YopH ${ }^{11}$ foram testadas com soro de coelho anti-YopH ${ }^{11}$, soros de coelhos imunizados com as cepas de $Y$. pestis e soros humanos com suspeita clínica de peste (HA-).

\section{RESULTADOS E DISCUSSÃO}

A morbimortalidade por peste entrou em declínio nos focos brasileiros a partir da década de 70, a atividade pestosa continuou a ser detectada nos inquéritos, mas os casos notificados nos últimos anos, apesar de sua fundamentação clínico-epidemiológica, têm sido descartados por se apresentarem reiteradamente negativos à pesquisa de anticorpos contra 0 antígeno $\mathrm{F} 1 \mathrm{da} Y$. pestis pela НА.

Estes resultados poderiam ser atribuídos a uma baixa sensibilidade da técnica, falta de resposta ao antígeno F1, erro no diagnóstico clínico-epidemiológico e até à coleta e transporte inadequados de amostras ${ }^{40}$. Para verificar a validade destas hipóteses 278 amostras de soros obtidas de pacientes suspeitos de peste na rotina do PCP-CE, que foram negativas à HA foram reexaminadas por Dot-Elisa ${ }^{2}$ e, apesar da melhor sensibilidade do teste, os resultados permaneceram inalterados. Resultados semelhantes foram obtidos no DVBID/CDC com 15 amostras sorológicas (HA-) analisadas no SRP, que foram submetidas ao mesmo teste e a uma contraprova pelo método Competitive ELISA ${ }^{9}$.

A identificação de novos alvos para diagnóstico e o desenvolvimento de testes dirigidos a outros antígenos da Y.pestis contribuiria, para solucionar um eventual problema determinado por resultados falso-negativos que podem ocorrer com os testes tradicionais de detecção de anticorpos anti-F1. Assim sendo, a YopH, inicialmente denominada Yop51 em função do seu peso molecular ${ }^{7}$, por desempenhar importante papel na resistência bacteriana ao sistema imune do hospedeiro ${ }^{10}$ foi considerada uma boa candidata.

Uma proteína YopH recombinante com capacidade imunogênica em coelhos produzida por Costa ${ }^{11}$ favoreceu 0 desenvolvimento dessa pesquisa. Em trabalhos anteriores ${ }^{82122}$ foi observado que os soros de convalescentes de peste reconhecem as YopD e YopE e as Yops produzidas pelas três Yersinias patogênicas são reconhecidas por soros de animais imunizados com qualquer uma delas, comprovando a similaridade intra-específica dessas proteínas.

Em nossos ensaios de avaliação do potencial da proteína YopH houve reconhecimento da banda do tamanho correspondente ao da proteína YopH $(\sim 51 \mathrm{kd})$ apenas com o soro anti-YopH. Nos soros humanos houve reconhecimento de outras proteínas, mas não da YopH. Apesar da similaridade das Yops das Yersinias, a YopH recombinante não foi reconhecida pelos soros dos coelhos imunizados com cepas de $Y$. pestis. É possível que a falta de sinal 
no Western-blot com os soros dos coelhos anti-Y. pestis deva-se à baixa quantidade de anticorpos anti-YopH produzida frente à competição com outros epitopos mais imunogênicos, como o F1.

Os cinco soros de coelhos imunizados com as cepas brasileiras e estrangeiras de Y. pestis foram testados por Western-blot com o antígeno $\mathrm{F} 1$ e proteínas totais das cepas buscando identificar outras proteínas de interesse para o diagnóstico da peste. Todos os soros reconheceram a proteína $\mathrm{F} 1(17 \mathrm{kd})$. Nos extratos de proteínas totais, além dessa proteína, várias outras foram reconhecidas pelos soros, no entanto os seus padrões de reconhecimento nas diferentes cepas de $Y$. pestis foram muito variados e nenhuma proteína majoritária foi reconhecida por todos os soros, salvo a F1.

Um soro de coelho anti-F1 testado por Western-blot com o antígeno F1 e proteínas totais das cinco cepas de $Y$. pestis reconheceu tanto a proteína $\mathrm{F} 1$ purificada quanto uma outra de igual tamanho nos extratos de proteínas totais de todas as cepas, refletindo a similaridade da F1 produzida pelas cepas de diferentes focos do Brasil e de outros países. O soro de coelho anti-Y.pseudotuberculosis não mostrou reação com a membrana contendo F1, o que era previsível, pois ela não sintetiza esta proteína e, consequientemente, o soro do coelho infectado por tal bactéria não deve conter anticorpos contra este antígeno.

Dos 34 soros humanos testados com a F1 purificada, apenas os quatro soros positivos por HA reconheceram a proteína F1. Esses mesmos soros também reconheceram uma banda de tamanho da F1 nos extratos de proteínas totais das cepas de $Y$. pestis. Os soros HA- não reconheceram a F1 e os perfis obtidos com os diversos soros e as diferentes cepas foram muito variados.

A análise desses resultados permite concluir que as investigações epidemiológicas devem ser desenvolvidas por equipes multidisciplinares altamente qualificadas, que conheçam profundamente a epidemiologia regional e possam avaliar criteriosamente os casos suspeitos, reduzindo o número de falsos positivos e, conseqüentemente, a alta negatividade dos exames, o que permitirá o desencadeamento oportuno das medidas de controle enquanto se aguarda a confirmação laboratorial.

As equipes devem considerar todas as hipóteses no diagnóstico diferencial e não podem se restringir exclusivamente à obtenção de soro para a HA, devendo colher correta e sistematicamente amostras de sangue e, de acordo com o caso, aspirado de bubão, escarro e líquor céfalo-raquidiano (LCR) para a realização de exames bacteriológicos e moleculares. Por sua sensibilidade e especificidade, estes últimos devem ser aperfeiçoados visando a identificação de novos alvos e obtenção de técnicas que possam ser executadas nas diversas condições de uma investigação, enquanto os primeiros devem receber novamente prioridade na investigação, tendo em vista que o padrão-ouro no diagnóstico da peste continua sendo o isolamento da $Y$. pestis.

\section{AGRADECIMENTOS}

A Dra Jeannine Petersen do Diagnostic and Reference Laboratory, Bacterial Zoonoses Branch, Division of Vector-Borne Infectious Diseases, Centers for Disease Control and Prevention (DVBID/CDC, Fort Collins, CO/USA) pelas análises realizadas nas amostras sorológicas.

\section{REFERÊNCIAS}

1. Almeida AMP, Brasil DP, Leal NC, Melo MEB, Rego RVB, Almeida CR. Estudos bacteriológicos e sorológicos de um surto de peste no Estado da Paraíba, Brasil. Memórias do Instituto Oswaldo Cruz 84:249-256, 1989.

2. Almeida AMP, Ferreira LCS. Evaluation of three sorological tests for the detection of human plague in Northeast Brazil. Memórias do Instituto Oswaldo Cruz 87: 87-92, 1992.

3. Almeida AMP, Leal NC, Carvalho FG, Dantas-Sobrinho J, Almeida CR. Plague surveillance in Brazil: 1983-1992. Revista do Instituto de Medicina Tropical de São Paulo 37:511-516, 1995.

4. Almeida CR, Almeida, AMP, Vieira JB, Guida U, Butler T. Plague in Brazil during two years of bacteriological and serological surveillance. World Health Organization Bulletin 59:591-597, 1981.

5. Aragão AI, Seoane AC, Leal NC, Almeida AMP. Vigilância da peste no Estado do Ceará: 1990-1999. Revista da Sociedade Brasileira de Medicina Tropical 35:143$148,2002$.

6. Bahmanyar M, Cavanaugh DC. Plague Manual, World Health Organization, Geneve, 1976.

7. Bölin I, Portnoy DA, Wolf-Watz. Expression of the temperature-inducible outer membrane proteins of Yersiniae. Infection and Immunity 48:234-240, 1985.

8. Bölin I, Wolf-Watz. The plasmid-encoded Yop2 protein of Yersinia pseudotuberculosis is a virulence determinant regulated by calcium and temperature at the level of transcription. Molecular Microbiology 2:237-245, 1988.

9. Chu M. Laboratory Manual of Plague Diagnostic Tests, Centers for Disease Control and Prevention, World Health Organization, Geneve, 2000.

10. Cornelis GR. Molecular and cell biology aspects of plague. Proceedings of the National Academy of Sciences 97:8778-8783, 2002.

11. Costa SMR. Análise da expressão de proteinas fusionadas ao fator de virulência YopH de Yersinia enterocolitica sob o controle do promotor yopH em E. coli. Tese de Mestrado, Universidade Federal de Pernambuco, Recife, PE, 1999.

12. Fundação Nacional de Saúde. Peste. In: Guia de Vigilância Epidemiológica. $5^{a}$ edição. Ministério da Saúde, Brasília. Vol II, p.641-652, 2002.

13. Gage KL, Kosoy MY. Natural History of Plague: Perspectives from more than a century of research. Annual Review Entomology 50: 505-528, 2004.

14. Gomes-Solecki MJ, Savitt AG, Rowehl R, Glass JD, Bliska JB, Dattv. LcrV capture enzyme-linked immunosorbent assay for detection of Yersinia pestis from human samples. Clinical Diagnosis Laboratory Immunology 12:339-346, 2005.

15. Higgins R. Emerging or re-emerging bacterial zoonotic diseases: bartonellosis, leptospirosis, Lyme borreliosis, plague. Review Scientific Tecnology. Office of International Epizootics 23:569-581, 2004.

16. Hinnebush BJ, Rudolph AE, Cherepanov P, Dixon JE, Schwan TG, Forsberg A. Role of Yersinia murine toxin in survival of Yersinia pestis in the midgut of the flea vector. Science 296:733-735, 2002.

17. Mazza G, Karu AE, Kingsbury DT. Immune response to plasmid- and chromosomeencoded Yersinia antigens. Infection and Immunity 48:676-685, 1985.

18. Payne FE, Smadel JE, Courdurier J. Immunologic studies on persons residing in a plague area. Infection and Immunity 77:24-33, 1956.

19. Perry RD, Fetherston JD. Yersinia pestis - Etiologic agent of plague. Clinical Microbiology Reviews 10:35-66, 1997.

20. Sousa AQ, Parise ME, Pompeu ML, Coelho-Filho JM, Vasconcelos IAB, Lima JWO, Oliveira EG, Vasconcelos AW, David JR, Maguire JH. Bubonic leishmaniasis: a common manifestation of Leishmania (Viannia) brasiliensis infection in Ceara, Brazil. American Journal of Tropical Medicine and Hygiene 53:380-385, 1995.

21. Straley SC, Bowmer WS. Virulence genes regulated at the transcriptional level by $\mathrm{Ca}^{2+}$ in Yersinia pestis include structural genes for outer membrane proteins. Infection and Immunity 51:445-454, 1986.

22. Wolf-WatzH, Portnoy DA, Bölin I, Falkow S. Transfer of the virulence plasmid of Yersinia pestis to Yersinia pseudotuberculosis. Infection and Immunity 48:241-243, 1985.

23. World Health Organization. Technical Report Series, WHO Expert Committee on Plague, Fourth Report. World Health Organization Bulletin 447:23-25, 1970.

24. World Health Organization. Human plague in 2002 and 2003. Weekly Epidemiological Record 79:301-308, 2004. 